\title{
Comorbidity between Neurotic Depression and Post-Traumatic Stress Disorder (PTSD)
}

\author{
Huang Tao', Chunru Wang2", Juncheng Guo ${ }^{3}$, Min Guo ${ }^{1}$ \\ ${ }^{1}$ Hainan General Hospital, Haikou, China \\ ${ }^{2}$ People's Hospital of Qionghai City, Qionghai, China \\ ${ }^{3}$ Central South University Xiangya School of Medical Affiliated Haikou Hospital, Haikou, China \\ Email: *wchunru@163.com
}

How to cite this paper: Tao, H., Wang, C.R., Guo, J.C. and Guo, M. (2020) Comorbidity between Neurotic Depression and Post-Traumatic Stress Disorder (PTSD). World Journal of Neuroscience, 10, 135-140. https://doi.org/10.4236/wjns.2020.103015

Received: June 7, 2020

Accepted: August 4, 2020

Published: August 7, 2020

Copyright $\odot 2020$ by author(s) and Scientific Research Publishing Inc. This work is licensed under the Creative Commons Attribution International License (CC BY 4.0).

http://creativecommons.org/licenses/by/4.0/

(c) (i) Open Access

\begin{abstract}
Comorbidity with PTSD has been studied at home and abroad in follow-up studies following major traumatic events, indexing patients diagnosed with PTSD and investigating rates of comorbidity and other psychiatric disorders. In practical clinical practice, the majority of patients with PTSD are diagnosed with neurosis and depression, but the clinical manifestations and treatment of some of these patients are more complicated and difficult, and it is clinically found that these patients may have comorbidities and other mental disorders. This study in hospitalized patients with neurosis and depression as the main research object, through the investigation found that some patients (36.4\%) of comorbid PTSD, just, this part of the patients reported by a traumatic event is not CCMD-3 defined in the "unusual" traumatic event. Therefore, the results of this study suggest that attention should be paid to the collection of patients' medical history, especially the inquiry of traumatic experiences. This study is only a preliminary study with a small sample size, and the conclusions obtained need to be further confirmed in future studies.
\end{abstract}

\section{Keywords}

Neurosis, Depression, Comorbidities, Post-Traumatic Stress Disorder

\section{Introduction}

The concept comorbidity, first proposed by Professor Feinstein of Epidemiology at Yale University, United States, in 1970, was originally defined as "any disease other than the indexed disease under study that already exists or occurs in the process of indexing disease" [1], Comorbidity has received increasing attention in psychiatry since the introduction of the multi-axis diagnostic criteria for the 
Third Edition of the American Manual of Mental Disorders and Statistics (DSM-III) [2]. Post Traumatic Stress Disorder (PTSD) is a mental disorder with delayed appearance and long-term persistence caused by sudden, threatening or catastrophic life events [3]. Its main clinical manifestations are repeated invasive traumatic experience, continuous avoidance and increased alertness. Numerous studies of people with PTSD have shown that the rate of comorbidity with different mental disorders, including depression, anxiety, obsessive-compulsive disorder, and personality disorders, ranges from $10.0 \%$ to $79.4 \%$. However, there are few reports of comorbidity or PTSD among other mental disorders. The purpose of this study was to investigate the rates of comorbid PTSD among patients clinically diagnosed with neurosis and depression [4].

\section{Objects and Methods}

\subsection{Research Objects}

In march, 2019-December 2019 in Hainan province people's hospital psychological clinic and research in various cities and counties in Hainan province flow for clinical diagnosis of patients with neurosis and depression, into a set of standards: 1) Conform to the Chinese classification and diagnostic criteria for mental disorders third edition (CCMD-3) neurosis or depression diagnostic criteria (including the exclusion criteria); 2) The age range is 18 - 55 years old; 3) Primary school and above; 4) Stay in hospital for more than one week; 5) Willing to cooperate. A total of 160 cases were actually enrolled, including 85 males and 75 females.

\subsection{Research Methods}

After obtaining the "informed consent", the patients with neurosis and depression who met the enrollment criteria were investigated one by one with a self-made general condition questionnaire, including name, sex, age, marital status, family history, work status, etc. Screening was then performed using the Essener Trauma-Inventar (ETI) questionnaire [5]. There are 58 items in THE ETI, which are compiled by Tagay $\mathrm{S}$ et al. based on the diagnostic criteria for PTSD in the Fourth Edition of the Classification of Mental Disorders and Statistical Manual of Mental Disorders (DSM-IV), with high reliability and validity. The scale consists of five parts: the first part is the list of traumatic events, with 14 events. Participants were asked whether they had experienced a specific traumatic event (such as "natural disaster" or "rape"), and whether they had experienced it themselves or as a witness. Question 15 asked participants to write down other stressful life events not listed above. Participants were asked to pick the most stressful event, and the questions were all about the most stressful event. The second part consists of six questions about the most severe stress events, divided into two dimensions: objective description and subjective evaluation, corresponding to the DSM-IV diagnostic criteria respectively. Part THREE has 23 questions about current post-traumatic symptoms. It includes invasive 
symptoms, avoidance and increased alertness. A symptom score above 27 is considered a diagnosis of PTSD; a score between 16 and 26 is suspected; a score below 16 is not considered PTSD. Finally, 60 patients with ETI score of $>27$ were screened with post-traumatic Stress Syndrome 10-questions Inventory (ptss-10) for secondary symptom screening, and those with ptss-10 score of $b>35$ indicated obvious PTSD symptoms [6].

\subsection{Statistical Analysis}

Input the data into SPSS12.0 statistical software for Chi-square test.

\section{The Results}

1) Among 160 patients (85 males and 75 females) who met the enrollment criteria, 108 were clinically diagnosed as neurosis (32 cases of anxiety disorder, 22 cases of obsessive-compulsive disorder, 54 cases without clear classification); 52 cases of depression. There was no significant difference between the neurosis group and the depression group in gender, age, marital status, and work status $(\mathrm{P}>0.05)$ (Table 1).

2) Among all patients, there were 60 patients with ETI symptom score of $>27$, including 36 patients in neurosis group and 24 patients in depression group cases, accounting for $26.67 \%$ of the whole group; 36 patients with ETI symptom score $<26$ points and $>16$ points, and 31 patients with ETI symptom score $<16$ points cases; Among the 60 patients with ETI symptom score of $>27$, there were 33 cases with ptss-10 score of $>35$ (17 cases with neurosis, 16 cases of depression). The main traumatic events reported by the patients were neglect, abandonment and sexual assault in 10 cases 9 cases were committed, 9 cases were

Table 1. Comparison of general demographic data of 160 patients with neurosis and depression.

\begin{tabular}{ccccc}
\hline Project & $\begin{array}{c}\text { neuroticism } \\
(\mathrm{n}=108)\end{array}$ & $\begin{array}{c}\text { depression } \\
(\mathrm{n}=52)\end{array}$ & $\begin{array}{c}\text { Statistics } \\
X^{2}\end{array}$ & $\mathrm{P}$ \\
Gender & 57 & 28 & 0.016 & 0.899 \\
Male & 51 & 24 & & \\
Female & & & 0.642 & \\
Marriage & 56 & 29 & \\
Unmarried & 52 & 23 & & \\
Married & & & 0.649 \\
Working & 54 & 28 & \\
Are & 54 & 24 & & \\
No & & & & \\
Age & 62 & 27 & & \\
$18-35$ & 46 & 25 & & \\
$36-55$ & & & \\
\hline
\end{tabular}


divorced or separated, 8 cases were subjected to violent attacks, 5 cases were subjected to death of important relatives, and 3 cases were subjected to unexpected shock. 3 suffered from natural disasters, 4 suffered from physical and mental torture, 3 suffered from serious economic burden, 2 suffered from serious diseases, and the college entrance examination Failure: 4 case. The results of the two scales showed that all the 60 subjects had significant PTSD symptom groups, including "invasion", "enter", "avoid" and "increased alertness". And the second part of THE ETI shows that these traumatic events all lead to patient ownership.

The perception is one of severe helplessness and fear (Table 2).

\section{Discuss}

Different from previous studies indexed by PTSD, this study investigated the rate of comorbidities of neurosis, depression and PTSD among patients clinically diagnosed with neurosis and depression who were transferred to the department of Psychology of Hainan People's Hospital and the research team in all cities and counties of Hainan Province [7]. The results of this study showed that 40 of the 160 hospitalized patients with clinical diagnosis of neurosis and depression had comorbidities of PTSD, with a rate as high as $26.67 \%$. The 60 patients with ETI symptom score of $>27$ with ptss-10 were screened again, and 33 patients with ptss-10 score of $b>35$ were found, which showed good consistency between the two, indicating that this study was relatively reliable. Conclusion: Comorbid PTSD is not uncommon in patients with neurosis and depression [8]. The results also showed that the first onset time of 60 patients with ETI symptom score of $>27$ was similar to the reported traumatic event, suggesting that the onset of these 40 patients was closely related to the traumatic event [9]. Traumatic events can lead to neurosis and depression, but PTSD caused by traumatic events has not received enough attention. It is important to note that this study is based on neurosis and depression, which are both more severe and less effective in outpatient treatment. Therefore, the high rate of comorbidities PTSD in these patients does not mean that all patients with neurosis and depression have such a high rate of comorbidities PTSD [10]. In practical clinical work, neurosis, depression, comorbidities and PTSD are rarely diagnosed. For example, 40 patients with ETI score of $>27$ in this group were not diagnosed with PTSD at the same time. The reasons may be as follows: 1) Under the influence of the traditional principle of

Table 2. 160 Patients with ETI scale symptom score.

\begin{tabular}{cccccc}
\hline Project & $\begin{array}{c}\text { Neurotic } \\
(\mathrm{n}=54)\end{array}$ & $\begin{array}{c}\text { anxiety } \\
(\text { anxiety })\end{array}$ & $\begin{array}{c}\text { OCD } \\
(\mathrm{n}=22)\end{array}$ & $\begin{array}{c}\text { Depression } \\
(\mathrm{n}=52)\end{array}$ & $\begin{array}{c}\text { Total } \\
(\mathrm{n}=160)\end{array}$ \\
\hline ETI symptoms score $>27$ & 21 & 11 & 13 & 15 & 60 \\
16 ETI symptoms score $<26$ & 12 & 8 & 4 & 12 & 36 \\
ETI symptom score $<16$ & 11 & 8 & 3 & 9 & 31 \\
PTSS-10 score $>35$ & 10 & 5 & 2 & 16 & 33 \\
\hline
\end{tabular}


single diagnosis and hierarchical diagnosis, Chinese scholars often do not make comorbiditism diagnosis in the actual clinical work of psychiatric departments;

2) Ccmd-3 specifies that a traumatic event or situation (such as a natural or man-made disaster) that causes PTSD is a traumatic event or situation that is recognized as "unusual for everyone" [11]. But according to the DSM-IV criteria, the diagnosis of PTSD emphasizes both traumatic events and subjective feelings. This study shows that the traumatic events reported by a small number of patients are not unusual for everyone. For example, breakups and divorces are obviously not "exotic for everyone" traumatic events. But for some patients, these events mean being abandoned. Life is not settled and survival is threatened, which will lead to strong fear and helplessness in their subjective feelings. Therefore, such traumatic events can also lead to PTSD symptoms, and Chinese scholars under the influence of CCMD-3 will ignore such traumatic events and not diagnose PTSD. 3) Symptoms of PTSD were rarely cited as the main complaints of patients who came to see the doctor, such as depression, anxiety, restlessness, poor diet and sleep. "Avoidance" of traumatic events in the main symptoms of PTSD also causes the patient to avoid talking about traumatic events. The patient's avoidance attitude also causes doctors to not continue to ask whether there are traumatic events. The increased alertness of PTSD symptoms mainly manifests as difficulty in sleeping, or not sleeping deeply; irritated or easy to get angry. These symptoms are also the main clinical manifestations of neurosis and depression. 4) The clinical manifestations of the patients are not typical. In this study, 78 patients were clinically diagnosed with neurosis, among which only 20 were definitely diagnosed as "anxiety disorder" and "obsessive-compulsive disorder". It may be due to the atypical clinical symptoms of most neurosis patients in this group; the same patients may have a variety of clinical symptoms at the same time; especially in patients with comorbid PTSD, its clinical manifestation is more extensive; it is difficult to use only "anxiety" obsessive compulsive disorder diagnosis "to do" to sum up; so the clinical doctors sometimes are often only in accordance with the principle of single diagnosis dubbed "neurosis" diagnosis. The combination of these factors leads doctors to overlook the problem of comorbidities PTSD and to miss the diagnosis of comorbidities PTSD [12]. In this study, there were 45 cases in neurosis group, the ratio was $75.0 \%$; 15 cases in depression group, the ratio was $25.0 \%$, but the difference between the two groups was statistically significant, suggesting that the ratio of PTSD in neurosis group and depression group was different. However, the reason for the difference in the ratio is not clear at present. Maybe the relationship between emotional stability, PTSD and depression is cause and effect, which needs further study.

\section{Foundation Project}

Key Science and Technology Project of Hainan Province in 2018 (Approval Number: ZDYF2018140). 


\section{Conflicts of Interest}

The authors declare no conflicts of interest regarding the publication of this paper.

\section{References}

[1] Wittchen, H.U. and Essau, C.A. (1993) Comorbidity and Mixed Anxiety Depressive Disorder: Is There Epidemiologic Evidence. The Journal of Clinical Psychiatry, 54, 9-15.

[2] Svenaeus, F. (2014) Diagnosing Mental Disorders and Saving the Normal. Medicine, Health Care and Philosophy, 17, 214-244. https://doi.org/10.1007/s11019-013-9529-6

[3] Chen, C. and Gao, Z.-S. (2007) Comparative Study on Personality Characteristics and Social Support in Patients with Depression and Depression/Anxiety Comorbidities. International Journal of Internal Medicine, 34, 373-374.

[4] Keane, T.M. and Woife, J. (1990) Comorbidity in Post-Traumatic Stress Disorder An Analysis of Community and Clinical Studies. Journal of Applied Social Psychology, 20, 1559-1562. https://doi.org/10.1111/j.1559-1816.1990.tb01511.x

[5] Mehnert, A., et al. (2008) Psychological Comorbidity and Health-Related Quality of Life and Its Association with Awareness, Utilization, and Need for Psychosocial Support in a Cancer Register-Based Sample of Long-Term Breast Cancer Survivors. Journal of Psychosomatic Research, 64, 383-391. https://doi.org/10.1016/j.jpsychores.2007.12.005

[6] Guo, J.-C., Tian, Z.-L., Wang, X.-D., Guo, M., et al. (2016) Post-Traumatic Stress Disorder after Typhoon Disaster and Its Correlation with Platelet 5-HT Concentrations. Asian Pacific Journal of Tropical Medicine, 9, 890-892.

https://doi.org/10.1016/j.apitm.2016.07.011

[7] Bomyea, J., Risbrough, V. and Lang, A.J. (2012) A Consideration of Select Pre-Trauma Factors as Key Vulnerabilities in PTSD. Clinical Psychology Review, 32, 630-641. https://doi.org/10.1016/j.cpr.2012.06.008

[8] Bicanic, I.A., Postma, R.M., Sinnema, G., et al. (2013) Salivary Cortisol and Dehydroepiandrosterone Sulfate in Adolescent Rape Victims with Post Traumatic Stress Disorder. Psychoneuroendocrinology, 38, 408-415. https://doi.org/10.1016/j.psyneuen.2012.06.015

[9] Blier, P. (2013) Neurotransmitter Targeting in the Treatment of Depression. The Journal of Clinical Psychiatry, 74, 19-24. https://doi.org/10.4088/JCP.12084su1c.04

[10] Wu, S.W., Bu, T. and Hou, S. (2009) Advances in Genetic Research on Post-Traumatic Stress Disorder. Rehabilitation Theory and Theory in China Practice, 11, 1036-1037.

[11] Hegeman, J.M., Kok, R.M., Van der Mast, R.C., et al. (2012) Phenomenology of Depressionin Older Compared, with Younger Adults: Meta-Analysis. The British Journal of Psychiatry: The Journal of Mental Science, 200, 275-281. https://doi.org/10.1192/bjp.bp.111.095950

[12] Wang, Y. and Kasper, L.H. (2014) The Role of Microbiome in Central Nervous System Disorders. Brain, Behavior, and Immunity, 38, 1-12. https://doi.org/10.1016/j.bbi.2013.12.015 